Natura@economía

ISSN 2226-9479 (Versión electrónica) Website: http://revistas.lamolina.edu.pe/index.php/neu

\title{
Niveles de diversificación de ingresos económicos en hogares agropecuarios de la parroquia La Paz, Carchi, Ecuador
}

\section{Levels of diversification of economic income in agricultural households of the parish of La Paz, Carchi, Ecuador}

\author{
Karla Luna'; Marcelo Albuja ${ }^{2 *}$; Juan Pablo Aragón²; Fernando Basantes ${ }^{2}$ \\ ${ }^{1}$ Universidad Técnica del Norte. Universidad Técnica del Norte, Carrera de Ingeniería en Agronegocios, Avalúos y \\ Catastros. krafaela.moon@gmail.com \\ 2* Universidad Técnica del Norte, Facultad de Ingeniería en Ciencias Agropecuarias y Ambientales. Código Postal \\ 100105, Ibarra, Ecuador.1malbuja@utn.edu.ec
}

Recibido: 25 abril del 2019; Aceptado: 15 junio del 2019

\begin{abstract}
Resumen
El objetivo de la investigación fue analizar el grado de diversificación de ingreso de los hogares y proponer estrategias que disminuyan los niveles de pobreza. Para ello, se encuestó a 184 personas, se complementó con información bibliográfica, se aplicó la herramienta DAFO y la matriz CAME para el planteamiento de estrategias. Del estudio, se determinó que existen siete fuentes de ingreso en la parroquia, la principal es la agricultura con el $44 \%$ y el cultivo de papa es el rubro principal; el 54\% de productores han diversificado sus fuentes de ingresos, sin embargo el $56 \%$ del ingreso total del hogar lo generan las actividades fuera de finca. Como estrategias se propuso la comercialización asociativa y la creación de una ruta agroturística que contribuyan a incrementar los ingresos y mejorar la calidad de vida de los pequeños productores de la parroquia. Se concluye que, si los ingresos de las actividades agropecuarias no incrementan, las actividades fuera de finca seguirán ganando espacio lo que podría conllevar a pérdidas de producción de alimentos y migración del campo a la ciudad.
\end{abstract}

Palabras clave: Carchi; ingresos rurales; productividad; RIGA.

\begin{abstract}
The objective of the research was to analyze the degree of diversification of household income that allows proposing strategies to reduce poverty levels. A descriptive field research was carried out with the application of 184 surveys and was complemented with bibliographical research and the application of tools such as SWOT and the CAME matrix for the strategy approach. It was determined that there are seven sources of income in the parish, the main one is agriculture with $44 \%$ and the cultivation of potato as the main item, $54 \%$ of producers have diversified their sources of income, however $56 \%$ of the total income of the home is generated by off-farm activities. As strategies, associative marketing and the creation of an agrotourism route were proposed, contributing to increase income and improve the quality of life of the small producers of the parish. It is concluded that, if the income from agricultural activities does not increase, off-farm activities will continue to gain space, which could lead to losses in food production and migration from the countryside to the city
\end{abstract}

Keywords: Carchi; rural income; productivity; RIGA.

Forma de citar el artículo: Luna, K.; Albuja, M.; Aragón, J.P. 2019. Niveles de diversificación de ingresos económicos en hogares agropecuarios de la parroquia La Paz, Carchi, Ecuador. Natura@economía 4(1): 14 - 23 (2019). 


\section{Introducción}

De acuerdo a datos del Instituto Nacional de Estadísticas y Censos (INEC, 2019) la Encuesta Nacional de Empleo, Desempleo y Subempleo de Ecuador a diciembre de 2018, la pobreza por ingresos a nivel rural pasó de $39,3 \%$ a $40 \%$, una variación de $0,7 \%$ no estadísticamente significativa. No existen datos de la pobreza por ingresos en la parroquia La Paz; sin embargo, según las Necesidades Básicas Insatisfechas (NBI) la pobreza alcanzó un $82,8 \%$ al año 2015 . Para la Oficina Internacional de Trabajo (OIT, 2015) la diversificación de la economía rural tanto en las actividades agrícolas como no agrícolas, tiene un gran potencial para reducir la pobreza, aumentando los mecanismos de adaptación ante las malas cosechas o la volatilidad de los precios y, mejorando la seguridad alimentaria y de los medios de subsistencia de los hogares rurales.

Las actividades productivas de la parroquia La Paz son: agricultura, ganadería y silvicultura $(62,2 \%)$, comercio al por mayor $\mathrm{y}$ menor $(5,3 \%)$, industria y manufactura $(4,8 \%)$ transporte y almacenamiento $(3,8 \%)$ y construcción $(3,5 \%)$ como lo menciona el Plan de Ordenamiento y Desarrollo Territorial (2015). El principal problema que se identificó para la pobreza son los bajos ingresos que genera el sector agrícola por la baja productividad y concentración de la tierra (Casanova, 2011).

La economía rural puede clasificarse en: sector agrícola que produce materias primas, y sector no agrícola que comprende las actividades económicas no agrícolas que generan ingresos para los hogares rurales, es decir las diversas fuentes que se practican en el sector rural (OIT, 2015).

Las estrategias de obtención de ingresos se han relacionado con el estudio de pobreza en Latinoamérica, donde existe mayor índice de pobreza entre asalariados agrícolas que entre agricultores independientes, así como una menor incidencia en familias que han diversificado sus fuentes de ingresos (Quiñones, 2013).

En los estudios elaborados por CavigliaHarris \& Sills (2005) citado por Soriano (2015) sobre la diversificación agrícola, se encontró que tomar la decisión sobre diversificar puede impactar positiva $o$ negativamente en la distribución de ingresos en la familia. El impacto positivo se observa cuando por medio de la diversificación, las familias incrementan el nivel de ingresos, en consecuencia se abre una brecha de oportunidades para mejorar las condiciones de vida. De igual manera se menciona que en otros sectores la composición de ingresos se han diversificado incorporando salarios agrícolas y no agrícolas, transferencias gubernamentales y no gubernamentales, actividades no agropecuarias para mejorar su economía y por ende su condición de vida (Quiñones y Gálvez, 2015). Para los países en vías de desarrollo existe un proyecto llamado RIGA (Actividades Generadoras de Ingreso Rural) que tiene como objetivo principal promover el entendimiento del rol que dichas actividades juegan en la reducción de la pobreza y el fomento del desarrollo (Organización de las Naciones Unidas para la Alimentación y Agricultura, 2016). Los diferentes componentes de generación de ingresos del hogar rural son: ingresos por producción agrícola, producción pecuaria, empleo remunerado no agrícola, los negocios del hogar, empleo remunerado, las transferencias y otros ingresos no laborales.

El objetivo de la presente investigación fue analizar los niveles de diversificación de ingresos económicos de los hogares agropecuarios en la parroquia La Paz con la finalidad de proponer estrategias para el incremento de los ingresos económicos y el fortalecimiento del sector agropecuario, la seguridad y soberanía alimentaria.

\section{Materiales y métodos}

La presente investigación se realizó en la parroquia $\mathrm{La} \mathrm{Paz}$ en el cantón Montúfar, provincia del Carchi, Ecuador (Figura 1), conformada por nueve comunidades: San Francisco del Colorado, Cucher, Rumichaca, Pizán, Yail, Tuquer, Tesalia, Huaquer y la Gruta de La Paz, con una superficie aproximada de $112,166 \mathrm{~km}^{2}$, a una altitud entre los 2200 hasta los $3920 \mathrm{msnm}$ (Plan de Ordenamiento y Desarrollo Territorial, 2015).

Para determinar cuáles son las fuentes de ingreso de los pequeños productores agropecuarios de la parroquia $\mathrm{La} \mathrm{Paz}$ se realizó una investigación de campo con alcances exploratorios y descriptivos, se 
identificó a 351 pequeños productores, los mismos que son usuarios de los sistemas de riego que se encuentran habilitados jurídicamente y poseen extensiones de terreno entre 0 a 5 ha, se aplicó la formula respectiva para la obtención de la muestra, 184 productores quienes por medio de la técnica de la encuesta brindaron la información requerida.
Oportunidades) en la que se señala aspectos internos y externos de los pequeños productores agropecuarios de la parroquia La Paz, se aplicó la herramienta CAME (Corregir, Afrontar, Mantener, Explorar) la misma que ayudó a la formulación de posibles estrategias que contribuyan a mejorar los ingresos de los pequeños productores agropecuarios.

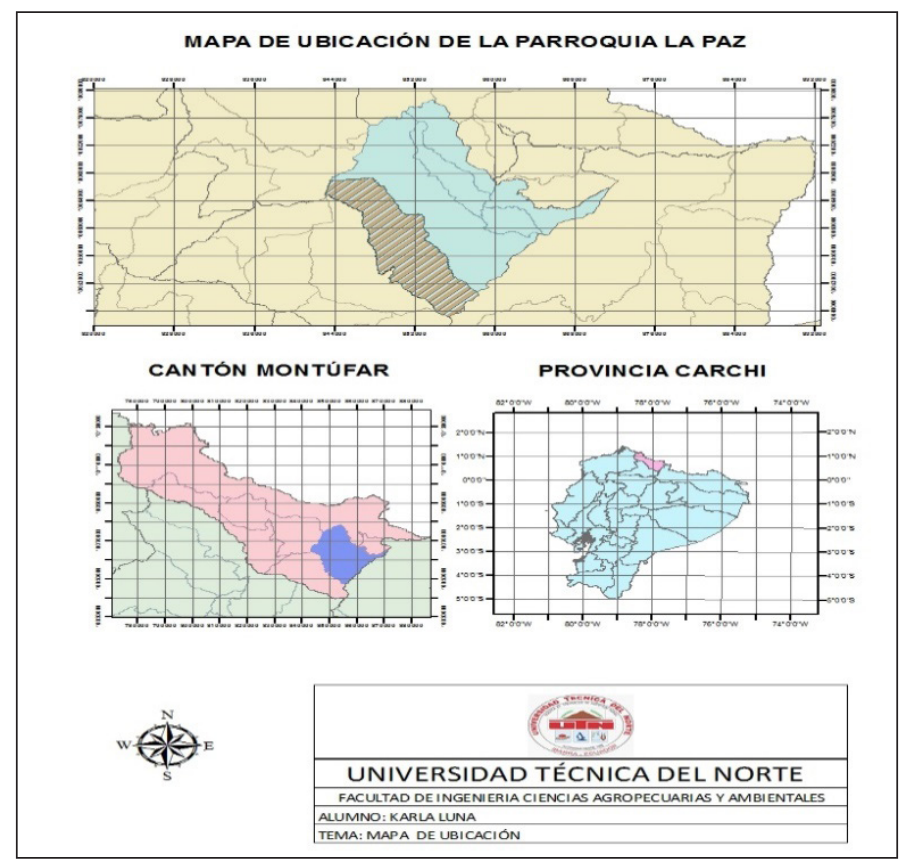

Figura 1: Ubicación de la parroquia La Paz, provincia del Carchi

Fuente: Instituto Geográfico Militar (2013)

El análisis de los resultados estuvo enfocado al proyecto RIGA y a la clasificación realizada por Carletto et al. (2007); Lanjouw (1998) citado por Báez y Castillo (2014) quienes mencionan que desde una perspectiva teórica un hogar rural obtiene ingresos por tres fuentes: I) por actividades agropecuarias; II) por actividades no agropecuarias y, III) por transferencias y otras fuentes. Se analizó y realizó la comparación con fuentes bibliográficas los costos de producción, el precio de venta y de las utilidades de la producción agrícola como pecuaria. Finalmente, con la matriz DAFO (Debilidades, Amenazas, Fortalezas,

\section{Resultados y discusión}

Del estudio se evidenció que los pequeños productores de la parroquia $\mathrm{La} \mathrm{Paz}$ desarrollan siete actividades para obtener ingresos económicos para sus hogares (Figura 2).

Es así que las actividades agropecuarias son desarrolladas por el $68 \%$ de hogares rurales, mientras que las actividades no agropecuarias representan $9 \%$ y como otra alternativa de ingresos el $23 \%$ de hogares reciben transferencias y otros ingresos.

En la Figura 3 se presenta el grado de diversificación de los hogares, entendiendo 
como diversificados aquellos donde además de las actividades agropecuarias tienen otra fuente de ingresos y no diversificados aquellos que solamente tienen ingresos agropecuarios.

La decisión de diversificar los ingresos en los hogares rurales suele basarse en factores incitadores, como la reducción del riesgo, la reacción a las crisis y la liquidez para estabilizar los flujos de ingresos, así como también la extensión de tierras que poseen los productores (OIT, 2015). que uno de los factores que influyen en la decisión de diversificación es la extensión de terreno, ya que con extensiones menores a 2 ha la diversificación es mayor y representa el $73 \%$ de pequeños productores, no así quienes poseen superficies de hasta 5 ha.

Es así que el Sistema de Investigación sobre la Problemática Agraria en Ecuador (2011) menciona que la producción campesina del Ecuador está marcada por la pequeña propiedad sobre la tierra, porque seis de cada 10 unidades productivas tienen

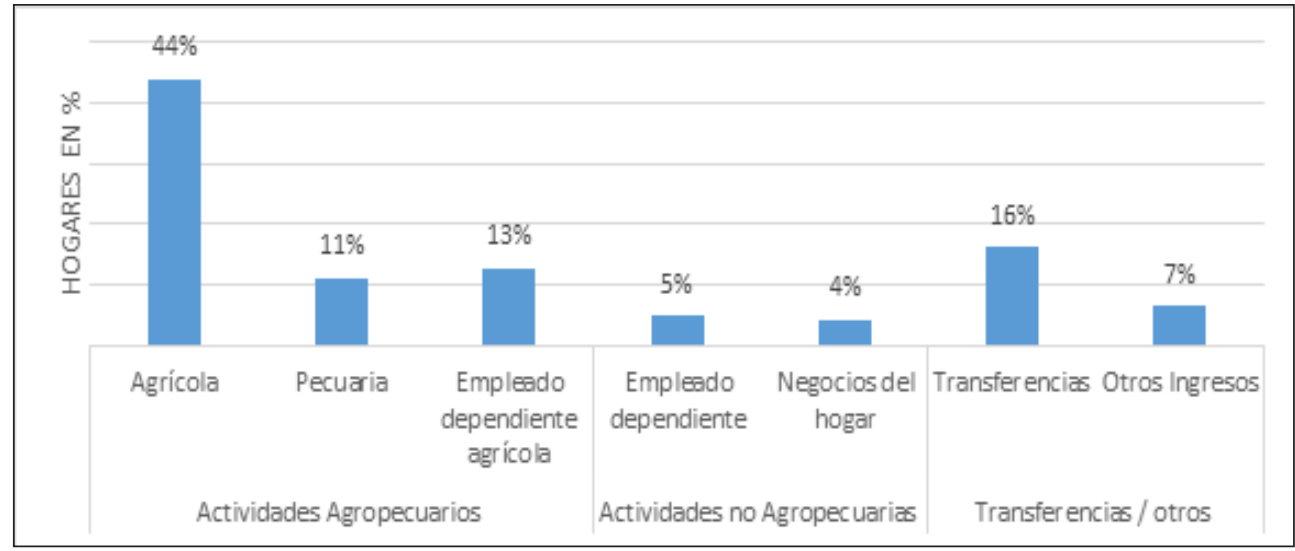

Figura 2. Actividades generadoras de ingresos rurales en la parroquia La Paz

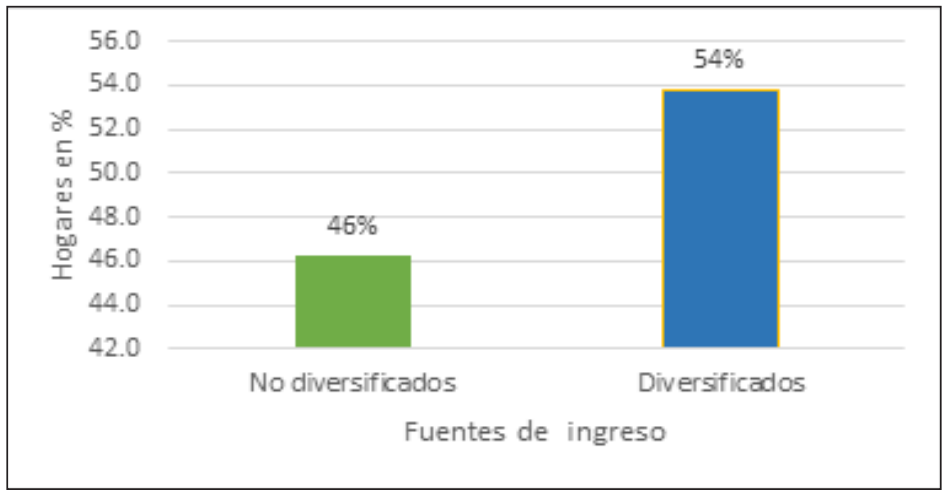

Figura 3. Diversificación de ingresos de los hogares rurales

En el presente estudio se refleja que el $54 \%$ de pequeños productores han diversificado sus ingresos realizando hasta tres actividades mientras que el $46 \%$ no diversifican ya que se dedican únicamente a la actividad agropecuaria, se puede mencionar una extensión menor a 5 ha. Por otro lado, la mitad de las familias rurales sobreviven en unidades productivas de dos a menos hectáreas. Esta estructura en el acceso a la tierra y a otros recursos naturales, acentúan el problema de la desnutrición, marginación, 
emigración y pobreza. Otro factor, es la diversificación en la edad del jefe de hogar y, los hogares que han optado por diversificar sus fuentes de ingresos han tenido resultados positivos debido al desarrollado de diferentes habilidades en torno a cada nueva actividad o por la estabilidad que estos ingresos les genera, especialmente en personas mayores de 65 años.
En la Figura 5 se hace un análisis de la composición de ingresos para determinar la importancia de cada una de las actividades que se desarrollan en la parroquia, así como el aporte que realizan al ingreso total de los hogares rurales.

Los resultados obtenidos reflejan que el $44 \%$ del ingreso total lo representa la actividad agropecuaria dentro de finca $y$

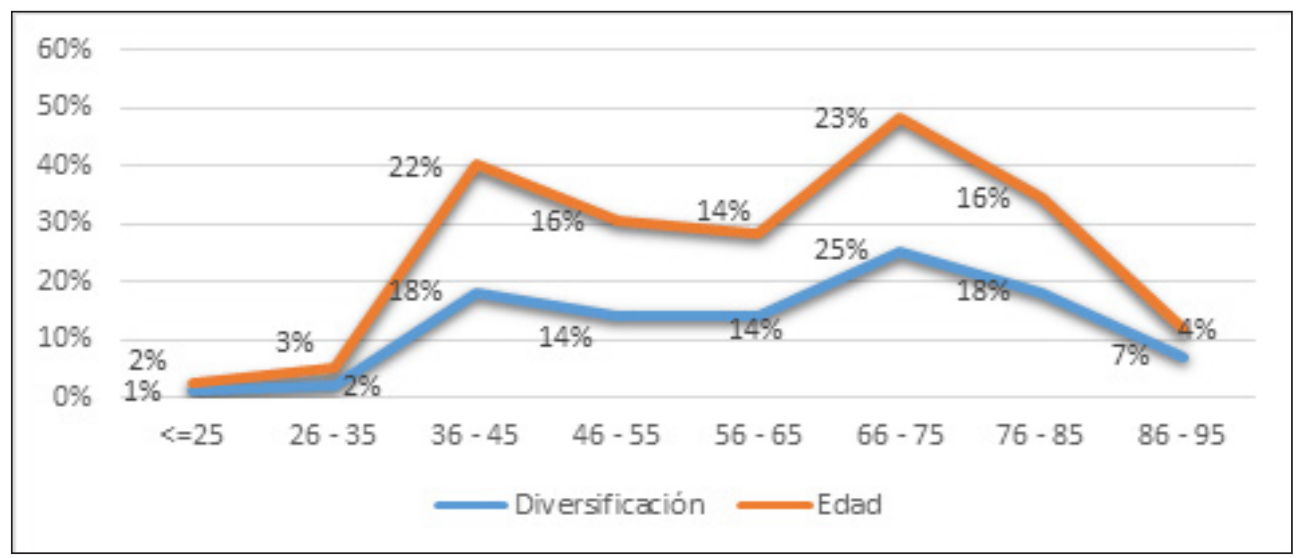

Figura 4. Diversificación de ingresos según la edad del jefe de hogar

La diversificación en función de la edad del jefe de hogar en esta parroquia obedece a los programas del Gobierno como es el caso del Bono de Desarrollo Humano, se puede observar que los resultados no coinciden con la literatura en jefes de hogar menores de 45 años. Se puede mencionar que existen un gran número de personas bajo dependencia, por cada 100 personas en edad óptima de trabajar, hay 64 en edad inactiva y hace referencia a los mayores de 65 años y menores de 15 años, concuerda con lo que menciona Quesada (2018) que el ámbito rural ha perdido a gran parte de sus efectivos en edad de trabajar al migrar a las ciudades. Eso provoca la falta de población fértil que genere un recambio generaciónal quedando personas de mayor edad en los núcleos poblacionales.

Es un hecho que el proceso de migración evidenciado es mayor por adolescentes para lo cual Suárez et al. (2012) en su revista señalan que la migración puede ser explicada por diferentes teorías socioeconómicas clásicas y/o recientes; ya que está ligado a aspectos culturales, económicos, sociales, financieros y emocionales. el $60 \%$ hacen mención que se encuentran en este grupo los empleados dependientes agrícolas, quienes optaron por diversificar sus ingresos y perciben un jornal o un ingreso diario a sus hogares.

Los ingresos de los hogares son bajos a pesar de que la actividad agropecuaria es la que mayor ingreso genera y la que mayor representatividad tiene en la parroquia, por el mayor porcentaje de pequeños productores agrícolas; sin embargo, no alcanzan a cumplir con el salario básico unificado, siendo el 55\% quienes anualmente perciben menos de 4000 dólares (Figura 6).

De igual manera se menciona que los pequeños productores agropecuarios no cubren con la canasta básica que presentó el INEC (2017), con un costo de $\$ 628$ para cuatro personas, que es el promedio de conformación del grupo familiar. Los resultados obtenidos en el presente estudio reflejan que el $82 \%$ de hogares están conformados por cuatro personas.

Según Burgos (2013) la pobreza por ingresos es un fenómeno que afecta asimétricamente a diferentes grupos 
humanos en especial a minorías étnicas y hogares cuyos jefes tienen características especiales (mujeres, mayores de 65 años e individuos no calificados); por ello, el nivel de instrucción es uno de los principales factores que influyen en la generación de ingresos.

El análisis hace relación a lo mencionado por Natera (2014) en su estudio, donde manifiesta que existe una relación positiva entre el nivel de instrucción y la capacidad de los individuos de insertarse en diferentes nichos del mercado laboral, en la parroquia La Paz el 58\% de los jefes de hogar han cursado únicamente la primaria, este es un impedimento para desarrollar actividades que generen mayores ingresos.

Los ingresos de la actividad agrícola y pecuaria se analizaron tomando en cuenta los principales cultivos del sector, rendimientos, costos de producción, ingresos, utilidad, riego, financiamiento y comercialización.

En la Figura 7 se representa los principales cultivos de la parroquia $\mathrm{La} \mathrm{Paz}$, que por diversidad de pisos climáticos da la oportunidad de obtener varios productos (Plan de Ordenamiento y Desarrollo Territorial, 2015).

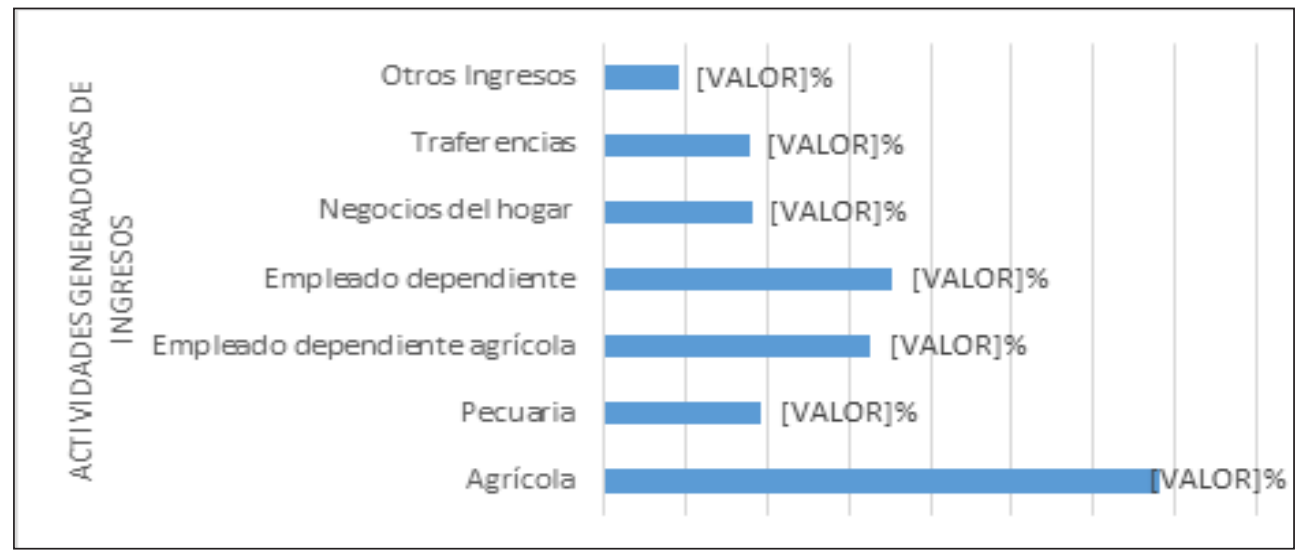

Figura 5. Composición de ingresos en promedio

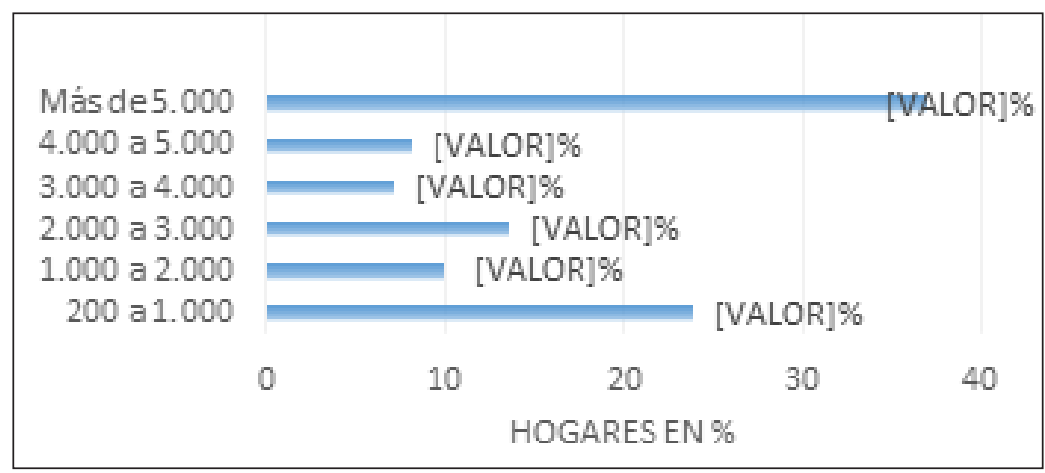

Figura 6. Ingreso anual de los hogares en dólares 


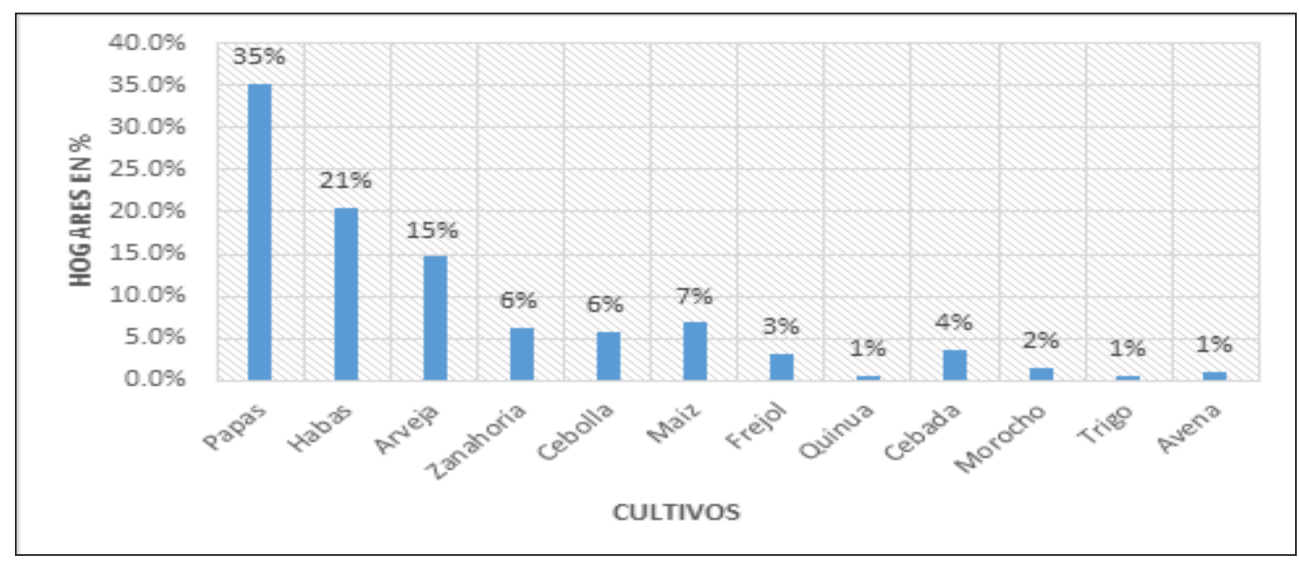

Figura 7. Principales cultivos de la parroquia La Paz

En su mayor porcentaje han optado por la producción de papas seguido por las habas, arveja, zanahoria, cebolla paiteña que dependiendo del mercado y precios de comercialización dejan grandes réditos a los productores y son cultivadas en su mayoría para recuperar los suelos y como rotación de cultivos para continuar con la producción de papa que en muchos casos genera la utilidad esperada y otros deja pérdidas totales, un menor porcentaje prefieren la producción de gramíneas que representan bajos costos de producción y gran utilidad.

Existen varios factores que influyen en la producción tanto agrícola como pecuaria en la que se encuentra; la semilla que se utiliza, los métodos de labranza, el clima tan variante, la utilización inadecuada y excesiva de químicos, la disponibilidad de agua de riego y la comercialización en la que no existe un control. En la Tabla 1 se detalla el rendimiento de los cultivos agrícolas (qq/ha) y el precio al que venden su producción, los mismos que se comparó con fuentes literarias principalmente del MAG (Ministerio de Agricultura y Ganadería), INIAP (Instituto Nacional de Investigaciones Agropecuarias), tesis, boletines agropecuarios y de situaciones entre otros.

Tanto los precios, la producción y la utilidad tienen cierta variación dependiendo del producto siendo un factor la falta de planificación de siembra, la aparición de enfermedades y plagas, sobreproducción, bajos precios de comercialización que son manejados por los intermediarios sin un control, el ingreso de productos de países vecinos a bajos precios y de forma ilegal, entre otros afectan directamente la economía de los pequeños productores agropecuarios, sus familias y por lo tanto de la comunidad.

En la Tabla 2, se refleja los productos que mayores ingresos generan a los pequeños productores de la parroquia La Paz así como los costos de producción, los ingresos y la utilidad que se obtuvo en el año 2017 de cada uno de ellos, la papa a pesar de ser el producto que se cultiva en mayor extensión, genera una utilidad similar que la cebolla paiteña ya que la inversión es mayor en cuanto a mano de obra y químicos, por otro lado las gramíneas (cebada, quinua y avena) tienen los costos de producción más bajos y su utilidad es significativa en los hogares rurales.

Se puede afirmar que los costos de producción de fuentes literarias son superiores a los declarados por los pequeños productores de la parroquia La Paz, ellos solo dan valor a la tierra cuando es arrendadas y representa el $10 \%$ del costo de producción total, en cuanto a la mano de obra en ciertos casos no es tomada en cuenta ya que son los mismos propietarios quienes realizan las labores, por otra parte los insumos químicos son los que mayor costo tienen ya que representan al $40 \%$ de la producción. 
Tabla 1. Comparación de rendimiento y precio de la producción agrícola

\begin{tabular}{cccc}
\hline Cultivo & $\begin{array}{c}\text { LA PAZ } \\
\text { P }(\text { USD }) \times \text { Q(qq/ha })\end{array}$ & $\begin{array}{c}\text { Otras fuentes } \\
\text { P }(\text { USD }) \times \text { Q }(q q / \text { ha })\end{array}$ \\
\hline Papas & $7 * 500$ & $10 * 450$ & SINAGAP * INEC-ESAG \\
Habas & $11 * 234$ & $10 * 165$ & SINAGAP * INEC-ESAG \\
Arveja & $24 * 151$ & $48 * 82$ & SINAGAP * INEC-ESAG \\
Cebada & $16 * 67$ & $20 * 54$ & TESIS * TESIS \\
Maíz & $18 * 32$ & $6 * 153$ & SINAGAP * INEC-ESAG \\
Zanahoria & $22 * 136$ & $12 * 250$ & SINAGAP * TELEGRAFO \\
Cebolla paiteña & $8 * 431$ & $12 * 800$ & SINAGAP * TESIS \\
Fréjol & $22 * 98$ & $28 * 101$ & SINAGAP * INEC-ESAG \\
Morocho & $42 * 35$ & $17 * 58$ & LÍDERES * LÍDERES \\
Quinua & $38 * 55$ & $80 * 15$ & EL COMERCIO * MAG \\
Avena & $30 * 40$ & $36 * 80$ & SINAGAP * INEC-ESAG \\
\hline
\end{tabular}

Tabla 2. Costos de producción, ingresos y utilidad por hectárea de los cultivos de la Parroquia

\begin{tabular}{ccccc}
\hline Cultivo & $\begin{array}{c}\text { Costos } \\
\text { (\$USD/ha }\end{array}$ & $\begin{array}{c}\text { Costos } \\
\text { Bibliografía }\end{array}$ & $\begin{array}{c}\text { Ingresos } \\
\text { (\$)USD/ha }\end{array}$ & $\begin{array}{c}\text { Utilidad } \\
(\$) \text { USD/ha }\end{array}$ \\
\hline Papas & 2652 & 3200 & 4862 & 2210 \\
Habas & 898 & 1385 & 2461 & 1563 \\
Arveja & 1333 & 1467 & 3645 & 2312 \\
Cebada & 205 & 600 & 1116 & 912 \\
Maíz & 479 & 742 & 579 & 100 \\
Zanahoria & 436 & 1200 & 927 & 491 \\
Cebolla paiteña & 1585 & 4500 & 3845 & 2260 \\
Fréjol & 780 & 1685 & 2154 & 1374 \\
Morocho & 500 & 1329 & 1500 & 1000 \\
Quinua & 1000 & 1297 & 2100 & 1100 \\
Avena & 250 & 1552 & 1200 & 950 \\
\hline
\end{tabular}

De igual manera es importante hacer un análisis de los costos en los que incurren los productores pecuarios basados en alimentación, establecimiento de espacios adecuados, costos de veterinario, transporte y otros, así mismo la utilidad que generan, la Tabla 3 representa la utilidad promedio que genera la actividad pecuaria.

Como lo menciona el Plan de Ordenamiento y Desarrollo Territorial, 2015 se ha identificado las actividades productivas de ganado y leche que son los dos ejes prioritarios que se desarrollan en la parroquia; es así que para el ganado vacuno, son 36 productores quienes poseen entre 1 a 27 cabezas de ganado, resaltando que se dedican a la crianza de vacas lecheras para obtener su producción y generar un ingreso extra; la producción porcina, la realizan 18 productores quienes tienen entre uno y 40 cerdos para diferentes propósitos como son el consumo familiar y la comercialización. Por otra parte, la producción de cuyes y aves en su mayor porcentaje son para el consumo familiar.

Tabla 3. Costo promedio de la producción pecuaria

\begin{tabular}{cccc}
\hline Tipo de animal & $\begin{array}{c}\text { Costo } \\
\text { en USD. }\end{array}$ & $\begin{array}{c}\text { Ingresos } \\
\text { en USD. }\end{array}$ & $\begin{array}{c}\text { Utilidad } \\
\text { en USD. }\end{array}$ \\
\hline Vacuno & 735 & 2500 & 1765 \\
Porcino & 1135 & 2075 & 940 \\
Cuyes/Conejos & 25 & 65 & 40 \\
Aves & 105 & 145 & 40 \\
\hline
\end{tabular}


En cuanto a los subproductos de la actividad pecuaria, los pequeños productores encuestados han optado por la obtención y comercialización de leche por la poca inversión. Son 19 pequeños productores que se dedican a la producción de leche con un promedio anual de 10947 litros, mismos que generan un ingreso de 3088 dólares anuales. Así mismo destinan para el consumo familiar de uno a dos litros diarios.

En base a los resultados obtenidos se formuló dos estrategias que buscan dinamizar la economía del pequeño productor; por lo tanto, de la comunidad en general, mejorar sus ingresos por medio de la comercialización asociativa de papa (principal producto de la parroquia) a través de la búsqueda de mercados, donde se garantice precios estables y justos de comercialización, así como también fortalecer las asociaciones existentes.

La siguiente estrategia, es la creación de una ruta agroturística, porque la parroquia La Paz cuenta con lugares turísticos religiosos de gran afluencia, como es la Gruta de la Virgen de la Paz, se sugiere establecer lugares estratégicos en cada una de las comunidades por las que atraviesa la ruta para comercializar en forma directa los diferentes productos agrícolas y dinamizar la actividad pecuaria ofertando platos típicos del sector, los pequeños productores van a desarrollar actividades de interacción y convivencia con los turistas.

\section{Conclusiones}

En los hogares de los pequeños productores de la parroquia la Paz se identificaron siete fuentes de ingreso siendo la principal la actividad agrícola (44\%); a pesar de ello, el $54 \%$ de productores han diversificado sus ingresos, reflejando que quienes poseen menos de dos hectáreas de terreno optan por desarrollar hasta tres actividades.

En cuanto a la representación en el ingreso total de los hogares rurales la actividad agrícola genera el mayor porcentaje $(34 \%)$, conjuntamente con la actividad pecuaria y empleado dependiente agrícola representan al 60\%; a pesar de ello, los hogares mantienen ingresos bajos ya que el $55 \%$ de hogares perciben anualmente 4000 dólares, es decir no alcanzan al salario básico mensual, mucho menos a cubrir los costos de la canasta básica, lo que ocasiona principalmente migración.

Los principales cultivos de la zona son: la papa, haba, zanahoria, arveja, cebolla paiteña, maíz, frejol y gramíneas, los cuales reflejan un bajo rendimiento que en comparación con fuentes bibliográficas debido tal vez a la limitada inversión, falta de planificación, uso inadecuado de químicos y por el precio bajo en la comercialización, la misma que se realiza a través de intermediarios.

Para la formulación de estrategias, se tomó en cuenta las fortalezas y oportunidades que se puede explotar como son el fortalecimiento de las asociaciones productivas mediante la comercialización asociativa en la que se reducen riesgos y se busca mercados estables con precios justos y fijos para su principal producto como es la papa, además una ruta agroturística en la que se busca dinamizar la economía de los pequeños productores de seis de las nueve comunidades de la parroquia, aprovechando su potencial turístico como es la gruta de la Virgen de La Paz.

\section{Literatura citada}

Baez, D. y Castillo R. 2014. Reducción de la pobreza rural en Ecuador. Un análisis de actividades generadoras de ingresos. Reporte de pobreza por consumo Ecuador 2006 - 2014. Disponible en: http://www.ecuadorencifras.gob.ec/ documentos/web-inec/Estudios\%20 e\%20Investigaciones/Pobreza_y_ desdigualdad/7.Reporte-Reduccion_de_ la_pobreza_rural_en_Ecuador.pdf

Burgos, S. 2013. Evolución de la pobreza y desigualdad de ingresos 2006 2012. Disponible en: http://www. planificacion.gob.ec/wp-content/uploads/ downloads $/ 2013 / 11 /$ Evoluci $\%$ C3\%B3nde - pobreza-y - desigualdad-deingresos-2006-2012_SBD_def.pdf

Casanova, G. 2011. Planificación Territorial. Obtenido de plan de desarrollo y ordenamiento territorial 2011-2031 del Cantón Montúfar.

Instituto Geográfico Militar. Disponible en: http://www.igmbolivia.gob.bo/

INEC [Instituto Nacional de Estadísticas y Censos]. 2017. ESPAC . Estadísticas Agropecuarias - ESAG: Instituto 
Nacional Autónomo de Investigaciones Agropecuarias. (2018). Información Técnica.

INEC [Instituto Nacional de Estadísticas y Censos]. 2019. Encuesta Nacional de Empleo, Desempleo y Subempleo en Ecuador. Obtenido de Estadísticas Nacionales.

Ministerio de Agricultura y Ganadería. 2018. Ministerio de Agricultura y Ganadería . Obtenido de Productividad Agrícola del Ecuador. Disponible en: http://sipa.agricultura.gob.ec/biblioteca/ rendimientos/Indice_productividad agrícola_2016.pdf

Natera, J. 2014. Nivel de instrucción de la población rural de la provincia de Tucumán. Un análisis intercensal. Universidad de Málaga.

OIT [Oficina Internacional del Trabajo]. 2015. Diversificación económica de la economía rural. Disponible en: http:// www.ilo.org/wcmsp5/groups/public/--ed emp/---emp policy/documents/ publication/wcms_437215.pdf

Organización de la Naciones Unidas para la Alimentación y la Agricultura. 2016. Clasificación de ingresos, base de datos. Disponible en: http://www.fao.org/ economic/riga/riga-database/es/

Plan de Ordenamiento y Desarrollo Territorial. 2015. Gobierno Autónomo Descentralizado Parroquia Rural de La Paz.

Quezada, A. 2018. El Telégrafo . Obtenido de La pobreza en la población rural : https://www.eltelegrafo.com.ec/noticias/ columnistas/15/la-pobreza-en-lapoblacion-rural

Quiñones, X. 2013. Tipos de estrategias de obtencion de ingresos y pobreza en familias mapuches rurales de Galvarino - Obtenido de http://www.scielo.org. ar/scielo.php? script $=$ sci arttext\&pid $=$ S1853-8665201300020002 1

Quiñones, X. \& Gálvez, J. 2015. Estructura de los ingresos de familias mapuches rurales de zonas periurbanas en Temuco, Chile. Mundo Agrario.

Sistema de Investigación sobre la Problemática Agraria en Ecuador. 2011. Atlas de la Tenencia de la Tierra en el Ecuador. Quito - Ecuador.
Soriano, M. 2015. Factores de diversificación en familias agrícolas (Tesis de grado) . México.

Suárez, F.; Cuenca, R. \& Hurtado, D. 2012. Incidencia de la migración familiar en el ámbito escolar y emocional en los niños/as de las provincias de Imbabura y Carchi-Ecuador. 